\title{
RIESGO DE LIXIVIACIÓN DE IMAZAPIR EN TRES SUELOS DE ARGENTINA
}

\section{RISK OF IMAZAPYR LEACHING IN THREE ARGENTINEAN SOILS}

\author{
Valeria Gianelli $^{1 *}$, y Francisco Bedmar ${ }^{2}$ \\ ${ }^{1}$ Instituto Nacional de Tecnología Agropecuaria, Estación Experimental Agropecuaria Balcarce, Ruta \\ 226 km 73.5, Balcarce, Argentina. gianelli.valeria@inta.gob.ar \\ 2 Universidad Nacional de Mar del Plata, Facultad de Ciencias Agrarias, Balcarce, Ruta 226 km 73.5, \\ Balcarce, Argentina. bedmar.francisco@inta.gob.ar \\ * Autor para correspondencia E-mail: gianelli.valeria@inta.gob.ar
}

\section{RESUMEN}

Imazapir es un herbicida de amplio espectro de control de malezas, perteneciente al grupo químico de las Imidazolinonas. En Argentina se aplica a gran escala en los cultivos de girasol y maíz Clearfield ${ }^{\circledR}$. Debido a que posee baja capacidad de adsorción, alta capacidad de desorción y prolongada persistencia en el suelo, representa alto riesgo de lixiviación y de contaminación del agua subterránea. En Argentina es escasa la información generada al respecto. Por lo tanto, el objetivo de este trabajo fue determinar el riesgo de lixiviación del herbicida imazapir hacia el agua subterránea en tres suelos contrastantes del país (Tandil, Anguil y Cerro Azul). Se utilizaron los índices GUS (Groundwater Ubiquity Score), Factor de Retardo (RF) y Factor de Atenuación log-transformado (AFT), considerándose para el cálculo de este último tres recargas netas de agua subterránea: 0,$3 ; 1,1$; y 2,3 mm día ${ }^{-1}$. Los índices fueron estimados empleando las propiedades ambientales (vida media y adsorción) de bases de datos y obtenidas experimentalmente para cada sitio de estudio. De acuerdo a los índices GUS y AFT, imazapir presentó alto riesgo de lixiviación hacia el agua subterránea en los tres suelos, según el orden Tandil > Cerro Azul > Anguil. Los resultados mostraron que una débil adsorción y una elevada persistencia podrían conducir a una alta movilidad de imazapir en el perfil del suelo, lo cual implicaría un alto riesgo de contaminación del agua subterránea.

Palabras clave: imazapir, contaminación, factor de retardo, factor de atenuación, GUS, propiedades ambientales

\section{ABSTRACT}

Imazapyr is a broad-spectrum herbicide that belongs to the imidazolinone chemical family. In Argentina it is used on Imidazolinone-tolerant corn and sunflower Clearfield ${ }^{\circledR}$. Due to its low adsorption capacity, and high desorption capacity and soil persistence, imazapyr is prone to leaching into groundwater. There is a lack of information available regarding groundwater leaching of imazapyr in Argentina. Therefore, the objective of this study was to determine the risk of imazapyr leaching in three Argentinean soils (Tandil, Anguil and Cerro Azul sites). The indexes applied to estimate lixiviation risk of the herbicide imazapyr were GUS (Groundwater Ubiquity Score), RF (Retardation Factor) and AFT (Attenuation Factor log-transformed). The indexes were calculated 
using environmental properties (half-life and adsorption) obtained from batch experiments, and from pesticides databases. AFT was calculated for three rates of net groundwater recharges: 0.3, 1.1 and $2.3 \mathrm{~mm} \mathrm{day}^{-1}$. According to the GUS and AFT indexes, imazapyr has a high leaching potential, following the order Tandil > Cerro Azul > Anguil soils. The results indicate that the weak adsorption and high persistence of imazapyr may increase its movement in the soil profile and the risk of groundwater pollution.

Key words: imazapyr, pollution, retardation factor, attenuation factor, GUS, environmental properties

\section{INTRODUCCIÓN}

Imazapir es un herbicida inhibidor de la enzima acetolactato sintasa del grupo químico Imidazolinona, de amplio espectro de control. Actualmente se utiliza a gran escala en Argentina, para el control de malezas en el cultivo de girasol (Helianthus annuus) y maíz (Zea mays) Clearfield (CL) tolerante a Imidazolinonas.

El movimiento y la persistencia de este herbicida en el suelo se encuentran fuertemente influido por diversas propiedades, como $\mathrm{pH}$, contenido de arcilla y de materia orgánica (Refatti et al., 2014; Bundt et al., 2015).

Dada su baja capacidad de adsorción, alta capacidad de desorción, y elevada solubilidad en agua, imazapir podría presentar un elevado riesgo de lixiviación hacia el agua subterránea. En tal sentido, Raimundo-Raimundo et al. (2011), encontraron en un estudio de lixiviación que más del 99\% de imazapir aplicado a columnas de suelo se recuperaba en los lixiviados, implicando un alto riesgo de contaminación de las aguas subterráneas. En otro estudio Refatti et al. (2014) detectaron efectos fitotóxicos en el cultivo de arroz (Oryza sativa), debido a la lixiviación de imazapir en los suelos.

Sumado a su potencial de lixiviación, entre sus propiedades fisicoquímicas se destaca su elevada persistencia en el suelo, lo cual incrementa el riesgo potencial de contaminación. En tal sentido, diversos estudios han determinado la presencia de imazapir en agua superficial y subterránea. Michael y Neary (1993) y Bush et al. (1995), encontraron residuos de imazapir en agua subterránea en el suroeste de Estados Unidos. En la misma línea, Börjesson et al. (2004), encontraron residuos del herbicida en el agua subterránea, después de 8 años de la aplicación, presentando niveles de contaminación entre 0,16 y 0,89 $\mu \mathrm{g}$ $\mathrm{L}^{-1}$. Por su parte, Battaglin et al. (1998), en un estudio en ríos y arroyos de EE.UU., detectaron concentraciones de imazapir superiores a los límites permitidos en más del $5 \%$ de las muestras analizadas. Mulder y Schmidt (2011) realizaron un estudio de monitoreo en el estado de Montana, con el objeto de determinar el impacto potencial de los compuestos químicos en el agua superficial y subterránea, encontrando presencia de imazapir en 42 y $75 \%$ de las muestras de agua superficial y subterránea, respectivamente, a concentraciones inferiores al valor máximo permitido por la Unión Europea en el agua para consumo humano de $0,1 \mu \mathrm{g} \mathrm{L}^{-1}$ (Hamilton et al., 2003).

En Argentina se han realizado diversos estudios en laboratorio, a campo y mediante bioensayos con el objeto de determinar la persistencia de imazapir en el suelo y sus efectos fitotóxicos sobre cultivos de invierno y de verano (Porfiri y Montoya, 2008; Gianelli et al., 2011; Gianelli et al., 2014). No obstante, es escasa la información relacionada con el riesgo de lixiviación de esta molécula hacia el agua subterránea (Gianelli et al., 2010; Porfiri et al., 2015).

El potencial de lixiviación de un compuesto químico puede ser determinado directamente o estimarse indirectamente mediante modelos, índices o indicadores (Oliveira et al., 2001). Los programas de monitoreo constituyen una herramienta fundamental para detectar la presencia de plaguicidas en el agua subterránea. Sin embargo, debido al elevado costo y tiempo necesario para llevar a cabo dichas técnicas, en los últimos años se han desarrollado diversas metodologías basadas en índices e indicadores, las cuales estiman el potencial de lixiviación de los plaguicidas y el riesgo de impacto en el agua subterránea. Dicha información constituye una herramienta que puede ayudar en la toma de decisiones y/o de control para la selección de plaguicidas, estrategias de manejo de plagas asociadas a los cultivos y/o sistemas de producción que minimicen el riesgo de impacto ambiental.

Los índices más utilizados son, entre otros, DRASTIC (Aller et al., 1985), LEACH (Laskowski et al., 1982), y GUS (Gustafson, 1991). El índice GUS (Groundwater Ubiquity Score) es una herramienta ampliamente empleada para clasificar a los plaguicidas en función de su riesgo de lixiviación hacia el agua subterránea. Este índice relaciona la persistencia (vida media) y adsorción $\left(\mathrm{K}_{\mathrm{oc}}\right)$ de los compuestos químicos en el suelo (Kerle et al., 1996).

A diferencia de estos procedimientos que sólo incluyen parámetros hidrológicos o 
exclusivamente propiedades químicas de los plaguicidas, Rao et al. (1985) desarrollaron un modelo para determinar la contaminación potencial del agua subterránea basado en el riesgo de lixiviación de los plaguicidas a través del perfil del suelo. Este método ha sido frecuentemente utilizado por diversos autores (Giambelluca et al., 1996; Costa Paraíba y Spadotto, 2002; Sanderson y Lowe, 2002; Bernard et al., 2005; Kookana et al., 2005) e incluye propiedades del suelo (densidad aparente, contenido de carbono orgánico, contenido de humedad a capacidad de campo, profundidad del perfil), características climáticas (recarga neta de agua subterránea) y algunas propiedades de los plaguicidas en estudio (vida media y coeficiente de partición en carbono orgánico). El modelo establece la determinación de dos índices: Factor de Atenuación (AF) y Factor de Retardo (RF). EL AF estima la fracción de plaguicida aplicado a la superficie que se lixivia a través del perfil del suelo, en base a la profundidad del horizonte de suelo considerado, el contenido de agua a capacidad de campo, la recarga neta de agua subterránea, la vida media de los plaguicidas en el suelo. Factor de Retardo (RF) determina la movilidad de los plaguicidas $y$ es definido en base a la densidad aparente del suelo, el contenido de carbono orgánico edáfico, el coeficiente de partición en carbono orgánico y el contenido de humedad en el suelo a capacidad de campo.

Diversos autores (Díaz-Díaz, 1998; Natale et al., 2002; Spadotto, 2002; Bernard et al., 2005; Kookana, 2005; Gianelli et al., 2010; Bedmar et al., 2015) han calculado los índices AF y RF, sin embargo, salvo algunas excepciones (Díaz-Díaz, 1998; Bernard et al., 2005), la mayoría de los trabajos utiliza información de adsorción y degradación de plaguicidas obtenida de bases de datos internacionales. Tal situación puede conducir a la obtención de resultados erróneos, debido a que pueden encontrarse grandes diferencias respecto de aquella información adaptada de estudios con diferentes suelos, condiciones ambientales, uso de plaguicidas y sistemas de producción.

En este sentido, Bedmar et al. (2013) evaluaron el riesgo de lixiviación de Atrazina, Acetoclor y S-metolaclor en dos suelos de la provincia de Buenos Aires, determinando mayor riesgo cuando el cálculo de los índices se realizaba con índices de adsorción y persistencia de los herbicidas en el suelo proveniente de base de datos.

Por lo anterior y para mejorar la confiabilidad de los resultados, resulta de gran trascendencia obtener información bajo las condiciones ambientales locales de uso de los plaguicidas. En base a este contexto, el objetivo de este trabajo fue determinar el riesgo de lixiviación hacia el agua subterránea del herbicida Imazapir en tres suelos contrastantes de Argentina, mediante índices simples y complejos, empleando las propiedades ambientales (vida media y $\mathrm{K}_{\mathrm{d}}$ ) de bases de datos y obtenidas experimentalmente para cada sitio en estudio.

\section{MATERIALES Y MÉTODOS}

\section{Sitios de estudio}

En los estudios se utilizaron muestras de suelo de las localidades de Tandil, Anguil y Cerro Azul, correspondientes a las provincias argentinas de Buenos Aires, La Pampa y Misiones, respectivamente. En base a la clasificación taxonómica de suelos (USDA, 1999), los suelos de las localidades de Tandil y de Anguil pertenecen al orden Molisol, clasificándose en función de la distribución de partículas, de textura franco arcillo arenoso y franco, respectivamente. Por su parte, el suelo de Cerro Azul se incluyó en el orden Ultisol, presentando textura arcillosa.

Las localidades seleccionadas para la extracción de las muestras fueron determinadas en función de las propiedades fisicoquímicas de los suelos, tales como diferente contenido de carbono orgánico (CO), pH, capacidad de intercambio catiónico (CIC), entre otras.

La recolección de las muestras $(0$ a $15 \mathrm{~cm}$ de profundidad) se realizó con un muestreador de suelo, obteniendo una muestra compuesta de aproximadamente $15 \mathrm{~kg}$ de suelo. En la Tabla $1 \mathrm{se}$ presentan las propiedades físico-químicas de los suelos.

Las muestras fueron secadas a $30^{\circ} \mathrm{C}$, molidas y tamizadas en malla de $2 \mathrm{~mm}$. Posteriormente, se realizó su caracterización física y química según las siguientes metodologías:

a) Textura: se realizó por el método sucesivo de la pipeta para determinar las fracciones de los tamaños de partículas por deposición gravimétrica (Gee, 1986; Bauder, 1986).

b) Carbono orgánico (CO): se analizó mediante oxidación con ácido crómico (Walkley y Black, 1934).

c) $\mathrm{pH}$ : se midió con electrodo en una relación suelo: agua de 1:2.5.

d) Capacidad de intercambio catiónico (CIC): se determinó por la sustitución con acetato de amonio $1 \mathrm{M} \mathrm{y} \mathrm{pH} \mathrm{=} 7$ (Chapman, 1965).

g) La densidad aparente ( $d \mathrm{sp})$ y el contenido de humedad gravimétrica $(\theta \mathrm{g})$ se estimaron a partir de muestras secadas a $105^{\circ} \mathrm{C}$ durante 24 horas.

h) Retención de humedad: se estimó mediante ollas de presión, las cuales se utilizaron para medir la curva característica de humedad del suelo a tensiones de 33 y 1500 kPa (Klute; Dirksen, 1986). 
Tabla 1. Contenido de carbono orgánico (CO), distribución del tamaño de partículas (arena, limo y arcilla), $\mathrm{pH}$, capacidad de intercambio catiónico (CIC), densidad aparente $\left(\varrho_{\mathrm{d}}\right)$ y contenido de humedad a capacidad de campo $(\Theta \mathrm{cc})$ de los suelos de Tandil, Anguil y Cerro Azul, Argentina ${ }^{a}$.

Table 1. Organic carbon content (OC); percentages of sand, silt, and clay; $\mathrm{pH}$; cation exchange capacity (CEC); bulk density $\left(\varrho_{\mathrm{d}}\right)$; water content at field capacity $(\theta c c)$ of soils at Tandil, Anguil and Cerro Azul sites, Argentina ${ }^{a}$.

\begin{tabular}{|c|c|c|c|c|c|c|c|c|}
\hline Suelo & $\begin{array}{c}\mathrm{CO} \\
\left(\mathrm{g} 100 \mathrm{~g}^{-1}\right)\end{array}$ & $\begin{array}{c}\text { Arena } \\
(\%)\end{array}$ & $\begin{array}{l}\text { Limo } \\
(\%)\end{array}$ & $\begin{array}{c}\text { Arcilla } \\
(\%)\end{array}$ & $\mathrm{pH}$ & $\begin{array}{c}\text { CIC } \\
\left(\mathrm{cmol}_{\mathrm{c}} \mathrm{g}^{-1}\right)\end{array}$ & $\begin{array}{c}d \text { ap } \\
\left(\mathrm{kg} \mathrm{m}^{-3}\right)\end{array}$ & $\begin{array}{c}\Theta c c \\
\left(\mathrm{~m}^{3} \mathrm{~m}^{-3}\right)\end{array}$ \\
\hline & $3,4(0,1)^{a}$ & & & $27,4(1,0)$ & & 25 & & $0,19(0,4)$ \\
\hline & & 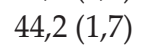 & & 2068 & & ) & & \\
\hline Cerro Azul & $2,5(0,1)$ & $5,4(0,7)$ & $16,7(0,3)$ & $78,0(1,0)$ & $4,9(0,1)$ & $20,6(0,5)$ & $1,1(0,01)$ & $0,25(0,6)$ \\
\hline
\end{tabular}

${ }^{a}$ Los valores reportados corresponden al promedio y desvío estándar de 4 repeticiones.

\section{Propiedades ambientales}

La persistencia del herbicida se determinó bajo condiciones controladas de laboratorio, obteniéndose la vida media $\left(\mathrm{T}_{1 / 2}\right)$ para cada suelo, mientras que la adsorción del herbicida se caracterizó a través de isotermas de adsorción, de las cuales se calculó el coeficiente de partición en adsorción $\left(\mathrm{K}_{\mathrm{d}}\right)$ para cada sitio de estudio (Gianelli et al., 2014). Las determinaciones del herbicida se llevaron a cabo mediante Cromatografía Líquida de Ultra Performance y Espectrometría de masas (UPLC- MS/MS) acorde a la metodología descripta en Gianelli et al. (2013).

Debido a que el carbono orgánico es el componente más importante del suelo, que determina la adsorción de los compuestos orgánicos neutros (Kah y Brown, 2006), se ha propuesto utilizar el $\mathrm{K}_{\mathrm{oc}}$ (coeficiente de partición en carbono orgánico) para determinar la adsorción de la mayoría de los plaguicidas. Claramente, este parámetro resulta poco eficaz para comparar la adsorción de compuestos iónicos como es el caso de imazapir. No obstante, cabe destacar que, para fines prácticos, los índices fueron estimados a partir de los $\mathrm{k}_{\mathrm{d}}$ y los valores de $\mathrm{K}_{\mathrm{oc}}$ de imazapir para cada uno de los suelos (Tabla 2).

Asimismo, se calcularon los índices empleando

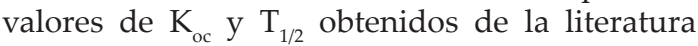
internacional con el fin de realizar comparaciones y determinar posibles diferencias.

Los valores de vida media y adsorción obtenidos experimentalmente para cada sitio y los reportados en la literatura internacional (AIDATA, 2016.) se presentan en la Tabla 2.

\section{Índices de lixiviación}

Para estimar el potencial de lixiviación de imazapir y el riesgo de impacto en el agua subterránea, se utilizaron los índices GUS (Groundwater Ubiquity Score) de Gustafson
(1991) y un modelo propuesto por Rao et al. (1985), el cual establece la determinación de dos índices: Factor de Atenuación (AF) y Factor de Retardo (RF). El factor de atenuación estima la fracción de plaguicida aplicado a la superficie que lixivia a través del perfil del suelo y se expresa a través de la siguiente ecuación:

$\mathrm{AF}=\exp \left[-\left(0,693^{*} \mathrm{~d}^{*} \theta_{\mathrm{FC}}{ }^{*} \mathrm{RF}\right) /\left(\mathrm{q}^{*} \mathrm{DT}_{50}\right)\right]$

donde $\mathrm{d}(\mathrm{cm})$ es la profundidad del suelo considerado, $\theta_{\mathrm{FC}}\left(\mathrm{m}^{3} \mathrm{~m}^{-3}\right)$ el contenido volumétrico de agua en el suelo a capacidad de campo, q $(\mathrm{cm}$ día $\left.^{-1}\right)$ la recarga neta de agua subterránea, $\mathrm{DT}_{50}$ (días) la vida media de los plaguicidas en el suelo y RF el factor de retardo.

En el presente trabajo se utilizó el índice AFT que corresponde a la transformación logarítmica del factor AF para simplificar la interpretación de los resultados (Bernard et al., 2005):

$\mathrm{AFT}=\mathrm{Ln}(\mathrm{AF}) /(-0.693)$

El factor de retardo indica la capacidad de los plaguicidas para lixiviar a través del suelo, teniendo en cuenta la adsorción y distribución entre las fases sólidas y líquidas y se define como:

$\mathrm{RF}=1+\left(\mathrm{Q}_{\mathrm{d}}{ }^{*} f_{\mathrm{oc}}{ }^{*} K_{\mathrm{oc}}\right) /\left(\theta_{\mathrm{FC}}\right)$

donde $\varrho_{d}\left(\mathrm{Mg} \mathrm{m}^{-3}\right)$ es la densidad aparente del suelo, $f_{\text {oc }}$ es la fracción decimal del contenido de C orgánico (Díaz-Díaz et al. 1998) y $K_{\mathrm{oc}}\left(\mathrm{L} \mathrm{kg}^{-1}\right)$ el coeficiente de reparto en $\mathrm{C}$ orgánico-agua.

En el presente estudio tanto el AF como el RF fueron calculados para cada suelo considerando sus propiedades fisicoquímicas y una profundidad de $15 \mathrm{~cm}$, a la cual fueron extraídas las muestras.

La recarga neta de agua subterránea se obtuvo 
Tabla 2. Vida media en el suelo $\left(\mathrm{T}_{1 / 2}\right)$ y coeficiente de partición en carbono orgánico $\left(\mathrm{K}_{\mathrm{oc}}\right)$ de imazapir obtenidos para cada suelo y valores reportados en base de datos.

Table 2. Soil half-life $\left(\mathrm{T}_{1 / 2}\right)$ and soil organic carbon sorption coefficient $\left(\mathrm{K}_{\mathrm{oc}}\right)$ obtained from batch experiments, and from pesticides databases.

\begin{tabular}{lll}
\hline Suelo & $\mathbf{T}_{\mathbf{1 / 2}}$ & $\mathbf{K}_{\mathbf{o c}}$ \\
\hline Tandil & 75 & 25 \\
Anguil & 37 & 41 \\
Cerro Azul & 121 & 85 \\
\hline Base de datos & 90 & 100 \\
\hline
\end{tabular}

a partir de la información calculada por Aparicio et al. (2008), quienes estimaron la recarga de la serie de suelos Mar del Plata por medio del modelo LEACHM, para una serie de 8 años, en un cultivo de maíz bajo riego suplementario. Dentro del período calculado, se seleccionaron los ciclos correspondientes a los valores de máxima $(2,3$ $\mathrm{mm}$ día $\left.^{-1}\right)$ y mínima $\left(0,3 \mathrm{~mm}\right.$ día $\left.^{-1}\right)$ recarga de agua, y el promedio de los 8 años $\left(1,1 \mathrm{~mm}\right.$ día $\left.^{-1}\right)$.

Asimismo, con el fin de corroborar estos valores, se realizó el cálculo de la recarga neta de agua, considerando el estudio realizado por Quiroz Londoño et al. (2012), quienes encontraron para el acuífero pampeano, valores de recarga entre 9 y 21\% del total de la precipitación, con un valor medio de $14 \%$. A tal fin, la recarga se determinó considerando el valor promedio correspondiente al $14 \%$ de las precipitaciones anuales para la localidad de Balcarce (período 1970-2011) obteniéndose un valor de 1,3 mm $\mathrm{d}^{-1}$ (Ecuación 4), valor similar al reportado por Aparicio et al. (2008) en su estudio.

$$
R=\frac{\sum P P}{\sum D P} * 14 \%
$$

donde $\mathrm{R}$ corresponde a la recarga neta $\left(\mathrm{mm} \mathrm{d}^{-1}\right)$, PP $(\mathrm{mm})$ al total de precipitaciones anuales y DP al número total de días con precipitaciones.

$\mathrm{Si}$ bien dichas valores fueron obtenidos por los mencionados autores, a nivel regional, en el presente estudio se consideraron los valores mencionados en forma estimativa, con el fin de calcular los índices y realizar comparaciones entre suelos, considerando valores de recarga neta de agua homogéneos entre los mismos.

Una vez obtenidos los índices RF y AFT, el herbicida se clasificó en base a su movilidad y su potencial de lixiviación empleando la clasificación propuesta por Khan y Liang (1989) (Tabla 3).

El índice GUS es considerado como indicador de la movilidad intrínseca de los plaguicidas, ya que consiste en una simple regresión empírica que incorpora solamente parámetros de adsorción y persistencia, para determinar el riesgo, y no incluye información de los suelos en estudio
(Spadotto, 2002).

El índice GUS es estimado mediante la siguiente ecuación:

GUS $=\log t_{1 / 2}\left(4-\log k_{O C}\right)$

Mediante este índice, los plaguicidas son clasificados como lixiviadores cuando el valor es $>2.8$, no lixiviadores si el índice es $<1,8$ y de transición cuando presenta valores entre 1,8 y 2,8.

\section{RESULTADOS Y DISCUSIÓN}

Los índices GUS, RF y AFT, fueron calculados para los suelos de Tandil, Anguil y Cerro Azul, considerando los valores del coeficiente de partición en adsorción normalizado por el contenido de carbono orgánico $\left(\mathrm{K}_{\mathrm{oc}}\right)$ y la vida media de imazapir, obtenidos a partir de los experimentos de persistencia y adsorción para cada uno de los suelos. Asimismo, para el cálculo del índice AFT se consideró la profundidad a la cual fueron extraídas las muestras de suelo (15 $\mathrm{cm})$.

Los valores calculados mediante el índice GUS para imazapir, y su clasificación correspondiente, en los suelos de Tandil, Anguil y Cerro Azul, se presentan en la Tabla 4.

De acuerdo al índice GUS, imazapir fue clasificado como lixiviable $(>2,8)$ tanto al utilizar valores de bases de datos, como al considerar las propiedades específicas de los suelos de Tandil, Anguil y Cerro Azul.

Si bien la categorización del herbicida fue la misma en los tres suelos estudiados, el herbicida presentaría mayor peligrosidad de contaminación según el orden Tandil > Cerro Azul > Anguil.

La degradación y adsorción de los herbicidas en el suelo controlan directamente el transporte de los compuestos hacia el agua subterránea. La tendencia establecida mediante el índice GUS es concordante con los resultados obtenidos en los experimentos previos. El suelo de Tandil presentó valores intermedios de persistencia y adsorción del herbicida, comparado con los 
Tabla 3. Clasificación de los índices Factor de retardo (RF), Factor de atenuación (AF) y Factor de atenuación ln-transformado (AFT) asociado a la movilidad y riesgo de contaminación.

Table 3. Classification of Attenuation Factor (AF), Retardation Factor (RF) and Attenuation Factor log-transformed (AFT) indexes related to mobility of the herbicide and risk pollution.

\begin{tabular}{|c|c|c|c|c|}
\hline RF & Clasificación & $\mathrm{AF}$ & AFT & Clasificación \\
\hline$=1$ & Muy móvil & $\geq 2,5 \times 10^{-1} y \leq 1$ & $\leq 2$ & Muy probable \\
\hline$>1 \mathrm{y}<2$ & Móvil & $\geq 1 \times 10^{-1} \mathrm{y}<2.5 \times 10^{-1}$ & $\geq 2 y<3$ & Probable \\
\hline$\geq 2 y<3$ & $\begin{array}{l}\text { Moderadamente } \\
\text { móvil }\end{array}$ & $\geq 1 \times 10^{-2} y<1 \times 10^{-1}$ & $\geq 3,3 y<7,2$ & $\begin{array}{l}\text { Moderadamente } \\
\text { probable }\end{array}$ \\
\hline$\geq 3 y<10$ & $\begin{array}{l}\text { Moderadamente } \\
\text { inmóvil }\end{array}$ & $\geq 1 \times 10^{-4} y<1 \times 10^{-2}$ & $\geq 7,2 y<13,3$ & Improbable \\
\hline$\geq 10$ & Muy inmóvil & $<1 \times 10^{-4}$ & $>13,3$ & Muy improbable \\
\hline
\end{tabular}

Tabla 4. Índice GUS y riesgo de lixiviación de imazapir en los suelos de Tandil, Anguil y Cerro Azul, considerando las propiedades ambientales (Koc y $\mathrm{T}^{1 / 2}$ ), obtenidos experimentalmente para cada suelo y de bases de datos.

Table 4. GUS index and leaching risk of imazapyr in Tandil, Anguil and Cerro Azul soils ${ }^{1}$, using environmental properties (Koc y $\mathrm{T}^{1 / 2}$ ), obtained from batch experiments and pesticides databases.

\begin{tabular}{lll}
\hline Suelo & Índice GUS & Clasificación \\
\hline Tandil & $4,9(0,04)$ & Lixiviador \\
Anguil & $3,7(0,11)$ & Lixiviador \\
Cerro Azul & $4,3(0,08)$ & Lixiviador \\
\hline Base de datos & 3,9 & Lixiviador \\
\hline
\end{tabular}

${ }^{1}$ Los números entre paréntesis corresponden al desvío estándar de cuatro repeticiones.

restantes suelos, por lo cual incrementaría el riesgo de lixiviación de la molécula. Por su parte en el suelo de Cerro Azul, si bien se registró mayor adsorción, lo cual limitaría la movilidad del herbicida en el perfil, este proceso también limitó posiblemente la disponibilidad para la degradación, incrementando en consecuencia el período de persistencia de la molécula y adicionalmente el riesgo de contaminación.

Los valores generados mediante los índices RF y AFT para imazapir, en los tres suelos estudiados, considerando tres valores de recarga neta $(q=0,3$; 1,$1 ; y 2,3 \mathrm{~mm} \mathrm{~d}^{-1}$ ) y los parámetros de persistencia y adsorción obtenidos experimentalmente se presentan en la Tabla 5.

En base al índice RF, el cual indica la movilidad relativa de los plaguicidas, imazapir categorizó como moderadamente inmóvil en Tandil y Anguil, y entre moderadamente inmóvil y muy inmóvil en Cerro Azul. Para su determinación, el mencionado índice utiliza parámetros de adsorción por lo cual la movilidad en los suelos siguió el orden Anguil $>$ Tandil $>$ Cerro Azul, producto de los valores de $\mathrm{K}_{\mathrm{d}}$ obtenidos experimentalmente en los estudios de adsorción.

En líneas generales, el potencial de lixiviación (AFT) de imazapir en los tres suelos, se incrementó con el aumento de la recarga neta de agua subterránea, siendo máximo a $2,3 \mathrm{~mm}$ día ${ }^{-1}$. El riesgo asociado al herbicida fluctuó entre improbable y muy probable, para las recargas de $0,3 \mathrm{~mm} \mathrm{~d}^{-1}$ y $2,3 \mathrm{~mm} \mathrm{~d}^{-1}$, respectivamente, presentando escasas variaciones entre los suelos. En tal sentido, considerando la recarga de $0,3 \mathrm{~mm}$ $\mathrm{d}^{-1}$, se determinó que la lixiviación de imazapir sería improbable en los tres suelos estudiados. No obstante, al seleccionar una recarga de 1,1 $\mathrm{mm}$ $\mathrm{d}^{-1}$, valor promedio obtenido para un período de 8 años, se determinó que el riesgo de lixiviación del herbicida osciló entre, probable en el suelo de Tandil y moderadamente probable en los suelos de Anguil y Cerro Azul. Finalmente, el riesgo se incrementó a muy probable en los tres suelos, cuando la recarga fue máxima $\left(2,3 \mathrm{~mm} \mathrm{~d}^{-1}\right)$.

La velocidad a la cual lixivian los plaguicidas a través de la zona no saturada o vadosa, y la proporción de plaguicida aplicado que alcanza el agua subterránea, está fuertemente relacionada al 
Tabla 5. Factor de Retardo (RF) y Factor de atenuación (AFT) calculados para imazapir en los suelos de Tandil, Anguil y Cerro Azul. Cálculos realizados para tres recargas, considerando las propiedades ambientales $\left(\mathrm{K}_{\text {oc y }} \mathrm{T}_{1 / 2}\right)$, obtenidos experimentalmente.

Table 5. Retardation Factor (RF) and Attenuation Factor log-transformed (AFT) calculated for imazapyr in Tandil, Anguil and Cerro Azul soils. Indexes were calculated for three rates of net groundwater recharges, using three environmental properties $\left(\mathrm{K}_{\mathrm{oc}} \mathrm{y} \mathrm{T}_{1_{1 / 2}}\right)$ obtained from batch experiments.

\begin{tabular}{lrrrr}
\hline \multirow{2}{*}{ Suelo } & \multirow{2}{*}{$\mathbf{R F}$} & \multicolumn{3}{c}{ AFT } \\
& & \multicolumn{1}{c}{$\mathbf{0 , 3}$} & $\mathbf{1 , 1}$ & $\mathbf{2 , 3}$ \\
\cline { 3 - 5 } & & $8,3(0,24)$ & $2,4(0,07)$ & $1,1(0,03)$ \\
Tandil & $6,5(0,12)^{1}$ & $11,8(0,94)$ & $3,4(0,27)$ & $1,5(0,12)$ \\
Anguil & $5,9(0,28)$ & $10,7(1,09)$ & $3,1(0,31)$ & $1,4(0,14)$ \\
Cerro Azul & $10,6(0,29)$ & &
\end{tabular}

${ }^{1}$ Los números entre paréntesis corresponden al desvío estándar de cuatro repeticiones.

flujo promedio de agua (recarga), así como a su variabilidad temporal (Giambelluca et al., 1996). Por tanto, los resultados del presente estudio, permitirían establecer que en años con abundantes precipitaciones o en situaciones de sobreriego se incrementaría el riesgo de lixiviación de imazapir y por ende la potencialidad de contaminación. Por el contrario, bajos volúmenes de drenaje aún con elevado contenido de plaguicidas en el suelo conllevarían a una reducción en las cantidades transportadas (Grignani y Laidlow, 2002).

En concordancia con el índice GUS y no obstante que la categorización del herbicida fue la misma en los tres suelos estudiados, excepto para la recarga de $1,1 \mathrm{~mm} \mathrm{~d}^{-1}$, si se consideran los valores numéricos, el herbicida presentaría mayor peligrosidad de contaminación en los suelos de Tandil y Cerro Azul, dado que el riesgo de lixiviación se incrementa progresivamente a medida que disminuyen los valores del índice AFT.

Por otra parte, se utilizaron valores de $\mathrm{K}_{\mathrm{oc}}$ y $\mathrm{T}_{1 / 2}$ obtenidos de la literatura internacional, para calcular los factores de Retardo y Atenuación a fin de cotejarlos con los calculados a partir de parámetros propios, obtenidos de los experimentos de persistencia y adsorción de este trabajo. Según los resultados, se registraron algunas diferencias sustanciales. En tal sentido, las categorizaciones de riesgo del herbicida fueron levemente inferiores respecto de la anterior forma de cálculo (Tabla 6). A modo de ejemplo, la movilidad de imazapir determinada mediante el RF, disminuyó, categorizando en este caso como muy inmóvil en los tres suelos. Asimismo el riesgo de lixiviación del herbicida (AFT) resultó menor para las tres recargas de agua consideradas. De esta manera, el riesgo de lixiviación pasó a ser muy improbable, y probable para los valores de mínima $\left(0,3 \mathrm{~mm} \mathrm{~d}^{-1}\right)$ y máxima recarga de agua $\left(2,3 \mathrm{~mm} \mathrm{~d}^{-1}\right)$, respectivamente. Por su parte, para la recarga de $1,1 \mathrm{~mm} \mathrm{~d}^{-1}$ categorizó como moderadamente probable en los suelos de Tandil y Cerro Azul y probable en el suelo de Anguil.

En base a lo expuesto, resulta evidente que los resultados obtenidos en la estimación del riesgo de lixiviación de los plaguicidas, pueden diferir según su forma de cálculo. Generalmente, para el cálculo de los estimadores de riesgo, se utiliza información de adsorción y degradación de plaguicidas obtenida de bases de datos internacionales. En tal sentido, esta aplicación podría resultar imprecisa para realizar determinaciones de riesgo sitio-específicas debido a que las interacciones plaguicidasuelo para un sitio individual no resultan bien representadas por la población de suelos incluidos en los estudios a partir de los cuales se genera la información (Green y Karickhoff, 1990). Por ello, es necesario obtener información de estas propiedades bajo las condiciones ambientales locales, debido a la estrecha relación que existe entre estos parámetros y los componentes de los suelos, que pueden afectar heterogéneamente el comportamiento de los plaguicidas.

En el presente estudio, imazapir presentó aproximadamente el mismo riesgo de lixiviación tanto con el índice GUS como con el Factor de Atenuación, sin embargo, es evidente que el modelo que considera al Factor de Atenuación y de Retardo, provee mayor información al incorporar propiedades de los suelos e hidrológicas, posibilitando de esta manera una mejor interpretación de los resultados.

Asimismo, los resultados obtenidos con los índices propuestos, fueron altamente coincidentes con la información generada experimentalmente en los estudios de persistencia y adsorción del herbicida. Los mismos indicarían que bajo las 
Tabla 6. Factor de Retardo (RF) y Factor de atenuación (AFT) calculados para Imazapir en los suelos de Tandil, Anguil y Cerro Azul. Cálculos realizados para tres recargas, considerando las propiedades ambientales $\left(\mathrm{K}_{\text {oc y }} \mathrm{T}_{1 / 2}\right)$, obtenidas de bases de datos.

Table 6. Retardation Factor (RF) and Attenuation Factor log-transformed (AFT) calculated for imazapyr in Tandil, Anguil and Cerro Azul soils. Indexes were calculated for three rates of net groundwater recharges, using environmental properties (Koc y $\mathrm{T}^{1 / 2}$ ) obtained from databases.

\begin{tabular}{lcccc}
\hline & & \multicolumn{3}{c}{ AFT } \\
Suelo & RF & \multicolumn{3}{c}{ Recarga $\left(\mathbf{m m ~ d}^{-\mathbf{1}}\right)$} \\
\cline { 3 - 5 } & & $\mathbf{0 , 3}$ & $\mathbf{1 , 1}$ & $\mathbf{2 , 3}$ \\
\hline Tandil & $23,1(0,96)^{1}$ & $24,5(0,16)$ & $7,1(0,54)$ & $3,2(0,07)$ \\
Anguil & $12,9(1,01)$ & $10,7(0,16)$ & $3,1(0,55)$ & $1,4(0,07)$ \\
Cerro Azul & $12,3(0,24)$ & $16,7(0,10)$ & $4,8(0,35)$ & $2,2(0,05)$ \\
\hline
\end{tabular}

${ }^{1}$ Los números entre paréntesis corresponden al desvío estándar de cuatro repeticiones.

condiciones del presente estudio, y considerando las propiedades del perfil del suelo, a $15 \mathrm{~cm}$ de profundidad el herbicida imazapir presentaría alto riesgo de lixiviación, siendo mayor el riesgo a medida que se incrementan los valores de recarga neta de agua subterránea. Esto coincide con Surgan et al. (2010) y Pfeiffer (2010), quienes informaron valores del índice GUS de 3,91, indicando en todos los casos un elevado potencial del herbicida para lixiviar y en consecuencia, un alto riesgo de contaminación del agua subterránea.

Asimismo, Montoya et al. (2010) emplearon el índice GUS para estimar la capacidad de lixiviación de imazapir en dos suelos de la provincia de La Pampa (Haplustoles Enticos), clasificando al herbicida como lixiviable. Por su parte, se han realizado diversos estudios previos, utilizando el Factor de Retardo y el Factor de Atenuación, en los cuales se determinó el riesgo de lixiviación de imazapir en suelos del sudeste de la provincia de Buenos Aires. En tal sentido Gianelli et al. (2008) evaluaron el riesgo de contaminación del agua subterránea con herbicidas aplicados en el cultivo de maíz, en la cuenca del Arroyo Pantanoso, Argentina, determinando que imazapir fue uno de los herbicidas con mayor riesgo de contaminación.

Sin embargo, Gianelli et al. (2010) determinaron el riesgo de lixiviación de imazapir en 18 series de suelo de la Cuenca del Arroyo Pantanoso, encontrando que el riesgo de lixiviación de imazapir resultó improbable. No obstante, en dicho trabajo para el cálculo de los índices se utilizó información proveniente de bases de datos internacionales y se consideró el perfil completo del suelo, por lo cual podría haberse subestimado el riesgo de contaminación del herbicida.

Estas aproximaciones sugeridas mediante los estimadores de riesgo han sido confirmadas por estudios de monitoreo, en los cuales se ha detectado la presencia de imazapir en el agua subterránea en diversas regiones del mundo (Michael y Neary, 1993; Bush et al., 1995; Börjesson et al., 2004; Mulder y Schmidt, 2011). Por lo tanto, los índices empleados en este trabajo permitirían establecer tendencias generales del riesgo potencial de lixiviación de este herbicida hacia el agua subterránea, constituyendo una herramienta eficaz para uso racional de los herbicidas, tendientes a minimizar su impacto negativo en el ambiente.

\section{CONCLUSIONES}

El riesgo potencial de contaminación del agua subterránea se incrementó en los suelos con mayor persistencia y adsorción del herbicida. El riesgo de lixiviación de imazapir en los diferentes suelos siguió el orden Tandil > Cerro Azul > Anguil.

De acuerdo al índice GUS imazapir presentó alto riesgo delixiviación en los tres suelos, mientras que el índice AFT indicó que la peligrosidad de contaminación del agua subterránea varió en función de la recarga neta del agua subterránea.

Los parámetros de adsorción y persistencia de imazapir obtenidos experimentalmente, permitieron realizar cálculos más confiables del potencial de contaminación del agua subterránea que la utilización de bases de datos.

Bajo las condiciones del presente estudio, imazapir presentaría alto riesgo de contaminación potencial del agua subterránea, siendo mayor a medida que se incrementan los valores de recarga neta del agua subterránea. 


\section{LITERATURA CITADA}

AIDATA. 2016. Pesticide Properties, Toxicities and Risk Ratings. Available at http://www. wsi.nrcs.usda.gov/ products/W2Q/ pest/ data/ AIDATA. Xls (Accessed 5 March 2016).

Aller, L.T., J. Bennett, R.J. Lehr, Y. Petty, and G. Hackett. 1985. DRASTIC: A Standardized System for Evaluating Ground Water Pollution Potential Using Hydrogeologic Settings. 622 p. U.S. Environmental Protection Agency. Report EPA/600/2-87/035. Washington D.C., USA.

Aparicio, V., J.L. Costa, y M. Zamora. 2008. Nitrate leaching assessment in a long-term experiment under supplementary irrigation in humid Argentina. Agric. Water. Manag. 95:1361-1372.

Battaglin, W.A., E.T. Furlong, M.R. Burkhardt, and J.C. Peter. 1998. Occurrence of Sulfonylurea, Sulfonamide, Imidazolinone, and other Herbicides in Midwestern Rivers, Reservoirs, and Ground Water. Sci. Total Environ. 248:123-133.

Bedmar, F., V. Gianelli, H. Angelini, y L. Viglianchino. 2015. Riesgo de contaminación del agua subterránea con plaguicidas en la cuenca del arroyo El Cardalito, Argentina. Rev. Investig. Agropecu. 41(1):70-82.

Bedmar, F., J.L. Costa, D. Gimenez, y P. Daniel. 2013. Comparación de dos métodos de obtención de índices para la estimación del riesgo de lixiviación de plaguicidas en dos perfiles de suelo. Agriscientia 30(2):69-78.

Bernard, H., P.F. Chabalier, J.L. Chopart, B. Legube, and M. Vauclin. 2005. Assessment of herbicide leaching risk in two tropical soils of Reunion Island (France). J. Environ. Qual. 34:534-543.

Börjesson, E., L. Torstensson, and J. Stenström. 2004. The fate of imazapyr in a Swedish railway embankment. Pest. Manag. Sci. 60(6):544-549.

Bundt, A.C., L. A Avila, A. Pivetta, D. Agostinetto. 2015. Imidazolinone degradation in soil in response to application history. Planta Daninha 33:341-349.

Costa Paraíba, L., and C.A. Spadotto. 2002. Soil temperature effecting calculating attenuation and retardation factors. Chemosphere 48:905-912.

Chapman, H.D. 1965. Cation exchange capacity. p. 891-900. In Black C.A. (ed.) Methods of soil analysis. Agronomy Series Number 9. American Society of Agronomy, Madison, Wisconsin, USA.
De Souza A.P., F.A. Ferreira, A.A. Da Silva, A.A. Cardoso, and A. Ruizh. 1999. Respiracao microbiana do solo sob doses de glyphosate e de imazapyr. Planta Daninha 1(3):387-398.

Díaz-Díaz R., J.E. García Hernández, and K. Loague. 1998. Leaching potentials of four pesticides used for bananas in the Canary Islands. J. Environ. Qual. 27:562-572.

Giambelluca T.W., K. Loague, R.E. Green, and M.A. Nullet. 1996. Uncertainty in recharge estimation: impact on groundwater vulnerability assessments for the Pearl Harbor Basin, O'ahu, Hawaii (USA). J. Contam. Hydrol. 23:85-112.

Gianelli, V., F. Bedmar, H. Angelini, V. Aparicio, y J.L. Costa. 2008. Riesgo de contaminación del agua subterránea con herbicidas aplicados en el cultivo de maíz en la Cuenca del Arroyo Pantanoso, Argentina. p. 59. En $2^{\circ}$ Congreso Argentino de la Sociedad de Toxicología y Química Ambiental (SETAC). 6o Reunión SETAC en Argentina. 26 al 28 de Noviembre de 2008, Mar del Plata, Argentina.

Gianelli, V., F. Bedmar, H. Angelini, V. Aparicio, y J.L. Costa, 2010. Determinación del riesgo de contaminación del agua subterránea con plaguicidas en la cuenca del Arroyo Pantanoso, Argentina. p. 135-152. En J.L. Fernández Turiel e I. González Hernández (eds.). Contaminación, Descontaminación y Restauración Ambiental. ISBN 978-84937437-1-0. Red Iberoamericana de Física y Química Ambiental, Salamanca, España.

Gianelli, V., F. Bedmar, y M.G. Monterubbianesi. 2011. Persistencia del herbicida Imazapir en el suelo y efectos fitotóxicos sobre cultivos de invierno y de verano. Rev. Investig. Agropecu. 37(1):18-25.

Gianelli, V, M.J. Zelaya, F. Bedmar, y J.L. Costa 2013. Determinación de residuos de imazapir en suelos de Argentina mediante cromatografía líquida de alta resolución y espectrometría de masas. Rev. Int. Contam. Ambient. 29(2):177-187.

Gianelli, V., F. Bedmar, and J.L. Costa. 2014. Persistence and sorption of Imazapyr in three Argentinean soils. Environ Toxicol Chem 33:129-34.

Gee, G.W., and J.W. Bauder. 1986. Particlesize analysis. p. 383-411. In Klute, A. (ed.) Methods of soil analysis. Part 1. Physical and mineralogical methods. Agronomy Series Number 9. Part 1. 2nd. ed. American Society of Agronomy and Soil Science Society of America, Madison, Wisconsin, USA. 
Green, R.E., y S.W. Karickhoff. 1990. Sorption estimates for modelling. p. 79-101. In Cheng H.H. (ed.) Pesticides in the soil environment: processes, impacts, and modeling. Book Series $N^{\circ}$ 2. Soil Science Society of America, Madison, Wisconsin, USA.

Grignani, C., and A.S. Laidlow. 2002. Nitrogen economy in grasslands and annual forage crops: control of environmental impact. $p$. 625-633. En Durand, J.L., Emile, J.C., Huyghe, C., Lemaire, G. (eds.) Proceedings of the 19th General Meeting of the European Grassland Federation on Multifunction Grasslands, Quality Forages, Animal Products and Landscapes. Vol. 7. British Grassland Society. Reading, UK, La Rochelle (F).

Gustafson, D.I. 1991. Groundwater ubiquity score: A simple method for assessing pesticide leachability. Envir. Sci. Tech. 8:339-357.

Hamilton, D.J, Á. Ambrus, R.M., Dieterle, A.S. Felsot, C.A. Harris, P.T Holland, et al. 2003. Regulatory limits for pesticide residues in water (IUPAC technical report). Pure Appl. Chem. 75(8):1123-1155.

Kah, M. and C.D. Brown. 2006. Adsorption of ionisable pesticides in soils. Reviews of Environmental Contamination and Toxicology 188:149-217.

Khan, M.A., and T. Liang. 1989. Mapping pesticide contamination potential. Environ. Manag. 13:233-242.

Kerle E.A., J.J. Jenkins, and P.A. Vogue. 2007. Understanding pesticide persistence and mobility for groundwater and surface water protection. 7 p. OSU Extension Service, EM 8561-E. Oregon State University, USA.

Klute, A., and C. Dirksen. 1986. Hydraulic conductivity and diffusivity: laboratory methods. p. 687-734. In Klute, A. (ed.) Methods of Soil Analysis. Part. 1. Agronomy No. 9. ASA and SSSA, Madison, Wisconsin, USA.

Kookana, R.S., R.I. Correll, and R.B. Miller. 2005. Pesticide impact rating index: a pesticide risk indicator for water quality. Water Air Soil Poll. 5:45-65.

Laskowski, D.A., C.A.I. Goring, P.J. Mccall, and R.L. Swann. 1982. Terrestrial environment. p.198-240. In Conway, R.A. (ed.) Environment Risk Analysis for Chemicals. Van Nostrand Reinhold Company, New York, USA.

Michael J.L., and D.G. Neary. 1993. Herbicide dissipation studies in southern forest ecosystems. Environ. Toxicol. Chem. 12:405410.
Montoya J.C., C. Porfiri, y M. Zelaya. 2010. Estimación de la adsorción de imazapir y atrazina al suelo y cálculo del Índice GUS. En 3ํㅡㄹ Congreso Argentino de la Sociedad de Toxicología y Química Ambiental (SETAC). 12-14 de Mayo de 2010. Santa Fe, Argentina.

Mulder, R., and C.H. Schmidt. 2011. Groundwater, surface water, and sediment monitoring for pesticides and nitrate in Billings, Montana. Montana Department of Agriculture, Helena, USA.

Natale, O.E., H. Allevato, M.C. Marzocca, y S. Sylvester. 2002. Evaluación de factores de riesgo debidos a plaguicidas en el medio ambiente rural. p. 68. En GTT5-programa de riesgos químicos-plan de gestión de sustancias químicas-Plan nacional de gestión de sustancias químicas. Ministerio de Salud y Acción Social, Ezeiza, Argentina.

Oliveira Jr. R.S., W.C. Koskinen, and F.A. Ferreira. 2001. Sorption and leaching potential of herbicides on Brazilian soils. Weed Res. 41:97-110.

Pfeiffer, M. 2010. Groundwater Ubiquity Score (GUS). Pesticides training resources. Available at http://www.ptrpest.com/pdf/ groundwater_ubiquity.pdf (Accessed 18 Janury 2016).

Porfiri, C., y J. Montoya. 2008. Persistencia de Imazapir y su efecto sobre cereales de invierno. p 43. En VII Congreso Nacional de Trigo. V Simposio Nacional de Cereales de Siembra Otoño-Invernal. Encuentro del Mercosur. Santa Rosa, La Pampa. Argentina.

Porfiri, C., J. Montoya., W. Koskinen, and P. Azcarate. 2015. Adsorption and transport of imazapyr through intact soil columns taken from two soils under two tillage systems. Geoderma 251-252:1-9.

Quiroz Londoño, O.M., D.E. Martinez, y H.E. Massone. 2012. Evaluación comparativa de métodos de cálculo de recarga en ambientes de llanura. La llanura interserrana bonaerense (Argentina), como caso de estudio. Dyna 171:239-247.

Raymundo-Raymundo, E. B. Prado-Pano, I. Nikolskii-Gavrilov, J. Mendoza-Hernández. 2011. Sorción, degradación y lixiviación de imazapyr en un suelo volcánico de México. Tecnología y Ciencias del Agua 2 (3):61-75.

Rao, P.S.C., A.G. Hornsby, and R.E. Jessup. 1985. Indices for ranking the potential for pesticide contamination of groundwater. Soil and Crop Science Society of Florida Proceedings $44: 1-8$. 
Refatti, J., L.A. Dirceu Agostinetto, R. ManicaBerto, A.C. Bundt, D. Balbé Elgueira. 2014. Efeito da calagem na lixiviação de imazethapyr e imazapyr em solo de cultivo de arroz irrigado. Ciência Rural, Santa Maria. 44(6):1008-1014.

Sanderson, I.D., and M. Lowe. 2002. Ground-water sensitivity and vulnerability to pesticides, Cache Valley, Cache County, Utah. p. 28. Utah Geological Survey, Washington D.C., USA.

Spadotto, C.A., M.A.F. Gomes, and A.G. Horsnby. 2002. Pesticide leaching potential assessment in multilayered soils. Pesticidas: Revista de Ecotoxicologia e Meio Ambiente 12:1-12.

Surgan, M., M. Condon, and C. Cox. 2010. Pesticide risk indicators: unidentified inert ingredients compromise their integrity and utility. Environ. Manag. 45:834-841.
USDA. 1999. Soil Taxonomy. A Basic System of Soil Classification for Making and Interpreting Soil Surveys. 871 p. Agriculture Handbook Number 436. United States Department of Agriculture; Natural Resources Conservation Service, 3333 U.S. Government Printing Office, Washington, D.C., USA.

Walkley, A., and A.I. Black 1934. An examination of the Degtjareff method for determining soil organic matter and a proposed modification of the chromic acid titration method. Soil Sci. 37:29-37. 